Schmerz 2007 $\cdot 21: 28-34$

DOI 10.1007/s00482-006-0497-0

Online publiziert: 17. August 2006

(c) electronic version (html)

Springer Medizin Verlag 2006

(c) print \& online pdf

Deutsche Gesellschaft zum Studium des Schmerzes 2006. Published by Springer Medizin Verlag - all rights reserved

G. Feigl ${ }^{1}$ F. Anderhuber ${ }^{2}$ J. H. D. Fasel ${ }^{1} \cdot$ R. Likar ${ }^{3}$

${ }^{1}$ Division für Anatomie im Department für Morphologie, CMU der Universität Genf

${ }^{2}$ Institut für Anatomie, Medizinische Universität, Graz

${ }^{3}$ Department für Anästhesiologie und Intensivmedizin, LKH, Klagenfurt

\title{
Die Bedeutung der Fascia stylopharyngea bei intraoralen Blockadetechniken
}

cessus styloideus (PS) und der lateralen Fläche der Pharynxwand (P) ausspannt (• Abb. 1). Sie trennt das Spatium parapharyngeum in eine vordere und hintere Abteilung. In Letzterer verlaufen große Gefäße und Nerven, wie die A. carotis interna (ACI), V. iugularis interna, N. glossopharyngeus (IX), N. vagus (X), N. accessorius (XI) und der Truncus sympathicus (TS) mit seinem GCS [10]. Die vordere Abteilung, u. a. mit Fettgewebe und der A. palatina ascendens gefüllt, weist eine Verbindung mit der Fossa infratemporalis auf, die den N. mandibularis $(\mathrm{V} / 3)$ beinhaltet. Nach unserem Kenntnisstand ist die kraniokaudale Ausdehnung der Faszie bisher nicht näher beschrieben worden. Weiter finden sich keinerlei Daten hinsichtlich der Durchgängigkeit dieser Bindegewebeplatte. Führt man nun die intraorale Technik der GLOA des GCS durch, könnte sich das Opioid bei den üblich verwendeten Injektionsvolumina von $2 \mathrm{ml}$ bei fehlender oder perforierter Faszie bis in die Fossa infratemporalis ausbreiten und den N. V/3 umspülen [7]. Letzteres würde zu dem sicherlich erwünschten Nebeneffekt der zusätzlichen Opioidwirkung an Schmerzfasern des N. V/3 führen. In dieser Arbeit wollen wir nun die genaue Anatomie dieser Faszie beschreiben. Von speziellem Interesse ist ihre Häufigkeit der Ausbildung und wenn vorhanden, ob sie teilweise oder voll die Abteilungen des Spatium parapharyngeums von der Fossa infratemporalis trennt und die Ergebnisse in Verbindung mit der
Erfolgsrate der Schmerzreduktion bei TN gebracht werden könnten.

\section{Material und Methode}

103 nach der Methode von Thiel [18] konservierte Kopfhälften (49 männliche [25 Köpfe zwischen 42 und 94 Jahre; Altersschnitt 75,32 Jahre]/54 weibliche Köpfe [27 Köpfe zwischen 41 und 101 Jahren; Altersschnitt 84,07 Jahre]) wurden untersucht. Von dieser Gesamtzahl wurden 10 unversehrte Kopfhälften und weitere 93 im Rahmen von Sezierübungen für höhersemestrige Humanmediziner seziert. Die 10 unversehrten Hälften wurden wie folgt untersucht. Von lateral wurden zuerst die Strukturen der Regio parotideomasseterica präpariert und anschließend entfernt. Nach Ablösen des M. masseter wurde die Mandibula (M) im Bereich des Korpus etwa fingerbreit vor dem Angulus mandibulae durchgeschnitten und die Fossa infratemporalis eröffnet. Die Kaumuskeln und die A. maxillaris samt Ästen wurden exstirpiert, die nervalen Inhaltsgebilde der Fossa infratemporalis und die laterale Wand des Pharynx $(\mathrm{P})$ präpariert. Anschließend wurde der Fettkörper vor der Faszie entfernt, die FSP vorsichtig aufgesucht und deren Vorderseite dargestellt. Entlang der A. carotis interna (ACI) wurde dann stumpf in das Spatium parapharyngeum eingegangen und die Faszienhinterwand abgehoben.

An den 93 Hälften im Rahmen der Sezierübungen vergewisserten wir uns vor 
Hier steht eine Anzeige.

第 Springer 

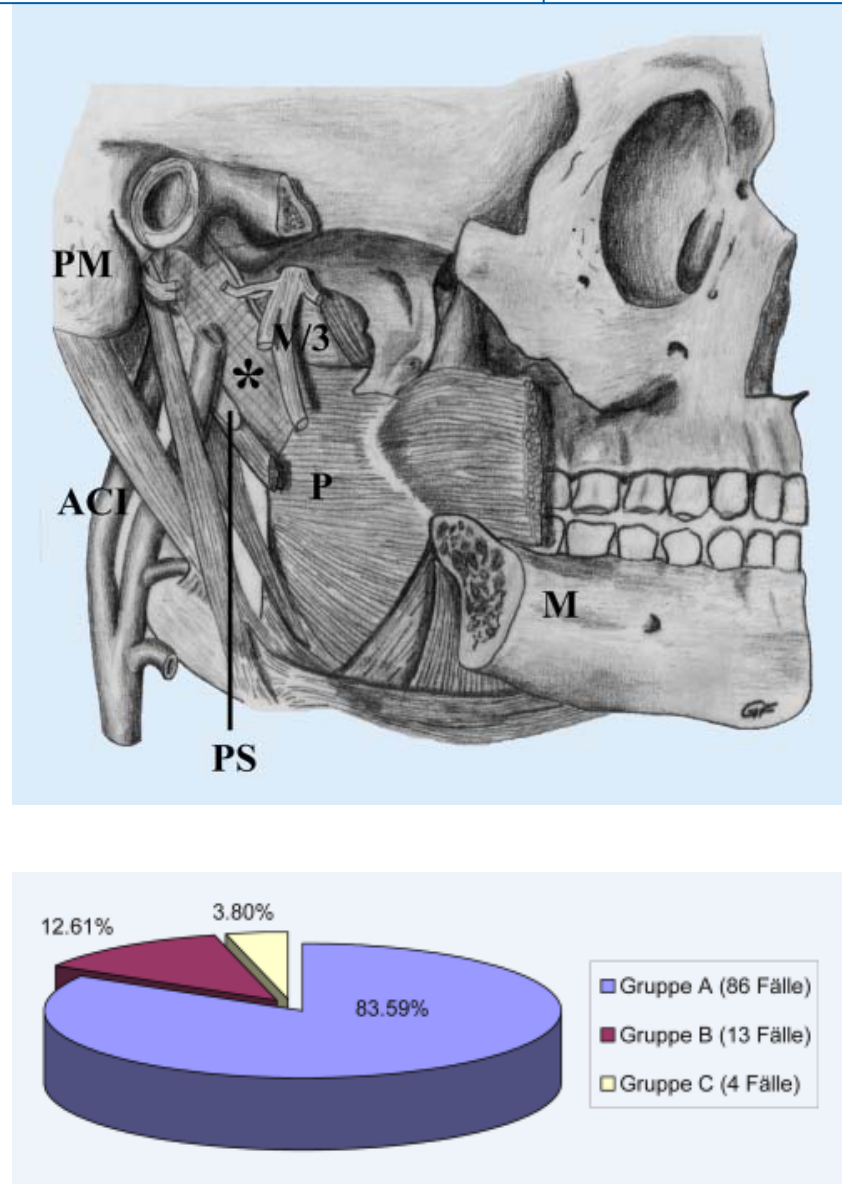

口Gruppe A (86 Fälle)

-Gruppe B (13 Fälle)

口Gruppe C (4 Fälle)
Abb. $1<$ Schematische Ansicht einer rechten Gesichtshälfte: Die Fossa infratemporalis ist eröffnet und der N. mandibularis (V/3) dargestellt. Die Fascia stylopharyngea (FSP, $\left.{ }^{*}\right)$ spannt sich zwischen Processus styloideus (PS) und Pharynxwand $(P)$ aus. Die A. carotis interna $(A C I)$ verschwindet hinter der Faszie im Spatium parapharyngeum. Als Orientierungspunkte sind die Mandibula (M) und der Processus mastoideus (PM) eingezeichnet. Die Abkürzungen bleiben in den Folgebildern gleich

Abb. $2 \triangleleft$ Ausbildung der Fascia stylopharyngea $(n=103)$

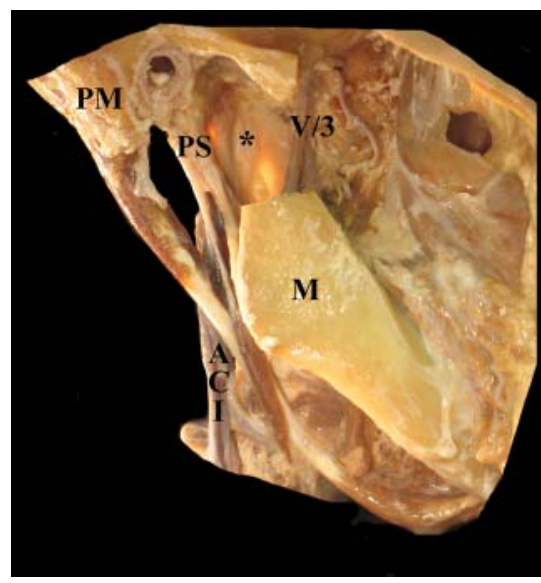

Abb. $3 \Delta$ Darstellung einer rechten Gesichtshälfte. Die Mandibula $(M)$ ist am Ramus mandibulae durchgeschnitten. Die Halswirbelsäule wurde in toto entfernt und die Faszie von hinten medial her beleuchtet. Die A. carotis interna $(A C l)$ verschwindet medial der FSP $\left({ }^{*}\right)$ und scheint ein wenig schattenhaft durch. Der N. $\mathrm{V} / 3$ teilt sich in der Fossa infratemporalis in seine Äste auf

der Untersuchung, ob die entscheidende Region nicht schon dargestellt worden war. Indiz für eine erhaltene Region war das Bestehen des Fettkörpers vor der Fas-

\section{Ergebnisse}

\section{Gruppe A}

In Gruppe A (• Abb. 2) war in 86 $(83,6 \%)$, davon 42 männlichen und 44 weiblichen Fällen, die Faszie vollständig ausgebildet (• Abb. 3 und 4). Sie breitete sich zwischen dem Processus styloideus (PS) und der lateralen Pharynxwand (P) aus. $\mathrm{Zu}$ beiden Seiten war sie sehr gut fixiert und konnte stumpf nicht gelöst werden. Kranial setzte sie stets an der Schädelbasis an. Der Ansatz verlief beginnend an der Basis des Processus styloideus (PS), anschließend an der Pars tympanica ossis temporalis dicht lateral vor dem Foramen jugulare und Canalis caroticus, medial der Fissurae petrotympanica und petrosquamosa bis zum Corpus ossis sphenoidalis und der Basis der Lamina medialis des Processus pterygoideus. Nach kaudal hin spannte sie sich zwischen Pharynx und den zum Riolan-Bouquet zählenden Muskeln bis auf Höhe des Angulus mandibulae aus. Dabei konvergierte sie nach kaudal hin und ergab die Form eines Dreiecks mit der Basis an der Schädelbasis. Damit war eine eindeutige Trennwand innerhalb vom Spatium parapharyngeum nach vorne hin gegeben. Die Faszie stellte sich in 1/3 der Fälle speziell im kaudalen Bereich als etwas schwächere Bindegewebeplatte dar, in den übrigen Fällen bot sie das Aussehen einer dichten Faszie mit teilweise aponeurotischem Charakter. Als Zusatzausbildung fanden wir in 10 Fällen eine 2., voll ausgespannte Bindegewebeplatte, die wir als Lig. sphenomandibulare (LSM) identifizieren konnten (• Abb. 4).

\section{Gruppe B}

In Gruppe B (• Abb. 2) war 13-mal $(12,6 \%)$ (3 männliche und 9 weibliche Hälften) die Faszie nicht vollständig ausgebildet. Sie bot mindestens eine Stelle der Perforation, wobei diese meistens im kaudalen Bereich zu finden war (• Abb. 5). Kranial dokumentierten wir auch in diesen Fällen eine dichte Bindegewebeplatte mit dem wie in Gruppe A beschriebenen Ansatz an der Schädelbasis. Präparate mit mehrfach durchgängigen Stellen fanden wir nur in 3 Fällen. 


\section{Gruppe C}

In Gruppe C (• Abb. 2) war in 4 Fällen $(3,8 \% ; 3$ männliche und ein weiblicher) keine Faszie ausgebildet. Die Sonde konnte ohne Probleme bis in die Fossa infratemporalis vorgeschoben werden (• Abb.6).

\section{Diskussion}

Der Erfolg peripherer Nervenblockaden hängt von zahlreichen Faktoren ab: Erfahrung des Anästhesisten, genauer Kenntnisse der Blockadetechnik, dem Wissen über die anatomischen Strukturen der betreffenden Regionen und ihrer Variationen. Wie sollen Anästhesisten aber gewisse, auf die Erfolgsrate Einfluss nehmende Strukturen kennen, wenn sie in der Literatur selten Erwähnung finden? Gerade bei diesen Problemstellungen gilt es, Abklärung mittels genauerer anatomischer Untersuchungen und literarischer Recherche zu leisten. Dies gilt besonders für die FSP. Sie ist in der aktuellen Literatur kaum vorhanden und findet sich eher in älteren deutsch- sowie französischsprachigen Lehrbüchern wieder. Weder die Terminologia Anatomica [6] noch sehr frequentiert benützte Anatomiebücher $[1,5,11]$ nennen diese Faszie. Eisler (in Lanz u. Wachsmuth [3]), repräsentativ für sehr umfassende Literatur, erwähnt die Faszie nur in einem Satz. Mehrere Zeilen finden sich nur bei Hafferl [10] und Frick u. Leonhardt [8]. Eine analoge Struktur zur FSP wird von Rouvière [14] als „Aileron du pharynx“, Flügelspitze des Pharynx, benannt. Schiebler [15] beschreibt die Faszie als „Aponeurosis stylopharyngea“, jedoch lassen sich auch hier keine Informationen bzgl. der kraniokaudalen Ausbreitung und der Ausbildung finden. Corning [2] weist auf dieses Bindegewebsblatt unter dem Namen „Fascia pharyngea“ hin, welche allerdings nicht mit der in Gray's Anatomy [1] benannten „pharyngeal fascia“ gleichzusetzen ist. In der englischsprachigen Literatur wird diese Faszie als Organfaszie des Pharynx interpretiert.

Von klinischem Interesse ist die Faszie bei der Therapie der TN, zu der die transorale GLOA des GCS herangezogen werden kann $[13,16,17]$. Dabei kann die

Schmerz 2007·21:28-34 DOI 10.1007/s00482-006-0497-0

(c) electronic version (html) Springer Medizin Verlag 2006

(c) print \& online pdf Deutsche Gesellschaft zum Studium des Schmerzes 2006

Published by Springer Medizin Verlag - all rights reserved

\section{G. Feigl·F. Anderhuber · J. H. D. Fasel · R. Likar \\ Die Bedeutung der Fascia stylopharyngea \\ bei intraoralen Blockadetechniken}

\section{Zusammenfassung}

Hintergrund. Mittels transoraler Blockade des Ganglion cervicale superius des Truncus sympathicus kann bei Trigeminusneuralgien durch Ausbreitung eines Opioids bis zum N. mandibularis eine zusätzliche Schmerzreduktion erzielt werden. Das Vorhandensein der Fascia stylopharyngea senkt möglicherweise die Erfolgsrate. Durch genaue anatomische Untersuchung der Faszie wollen wir auf die Effektivität der zusätzlichen Opioidwirkung rückschließen.

Material und Methode. 103 Kopfhälften wurden untersucht, die Faszie von lateral her aufgesucht. Dabei unterschieden wir 3 Gruppen: Gruppe A repräsentierte vollständig aus-

\section{Meaning of stylopharyngeal fascia in intraoral block techniques}

\section{Abstract}

Background. The almost unknown stylopharyngeal fascia may be one of the reasons for unsuccessful therapy of the trigeminal neuralgia by a transoral block of the superior ganglion of the sympathetic trunk. We investigated the anatomy of the fascia to show the efficiency of the block for this therapy.

Materials and methods. 103 halves of human heads were investigated. The stylopharyngeal fascia was dissected by a lateral approach. We classified three groups. Group A was formed by fascias without perforation, group $B$ by perforated fascias and group $C$ by all halves without a developed fascia. gebildete Faszienblätter, Gruppe B unvollständige Faszien und in Gruppe $C$ war die Faszie nicht ausgebildet.

Ergebnis. Die Faszie war in 86 vollständig und in 13 Fällen unvollständig ausgebildet. Lediglich in 4 Fällen fehlte sie vollends. Schlussfolgerung. Die Faszie kann die Erfolgsquote der Schmerzreduktion bei Trigeminusneuralgie beeinflussen.

\section{Schlüsselwörter}

Ganglionäre lokale Opioidanalgesie (GLOA) . Ganglion cervicale superius - Fascia stylopharyngea - Transorale Blockade · Trigeminusneuralgie

Results. We found a fully developed fascia in 86 cases. 13 halves had perforated fascias; in 4 cases the fascia was not developed. Conclusion. The stylopharyngeal fascia may inhibit the distribution of opoids into the infratemporal fossa associated with a consecutive block of the mandibular nerve and lower the rate of pain relief.

\section{Keywords}

Ganglionic local opioid analgesia (GLOA) . Superior cervical ganglion - Stylopharyngeal fascia - Transoral block - Trigeminal neuralgia 


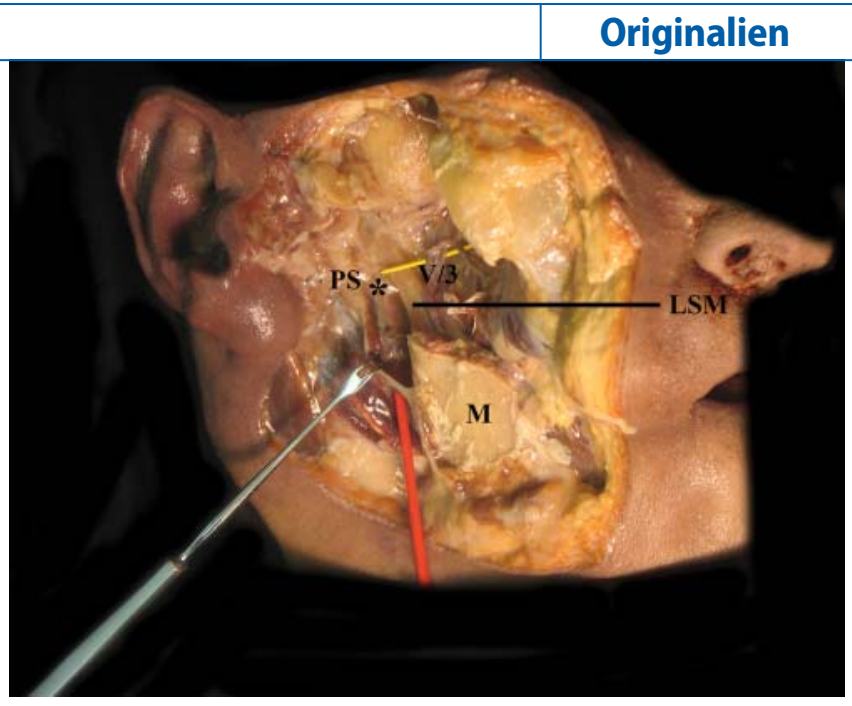

Abb. $4<$ Darstellung einer rechten Gesichtshälfte: Die FSP $\left.{ }^{*}\right)$ ist voll ausgebildet. Als Zusatzblatt spannt sich das Lig. sphenomandibulare (LSM) hinter dem gelb unterlegten N. $V / 3$ aus

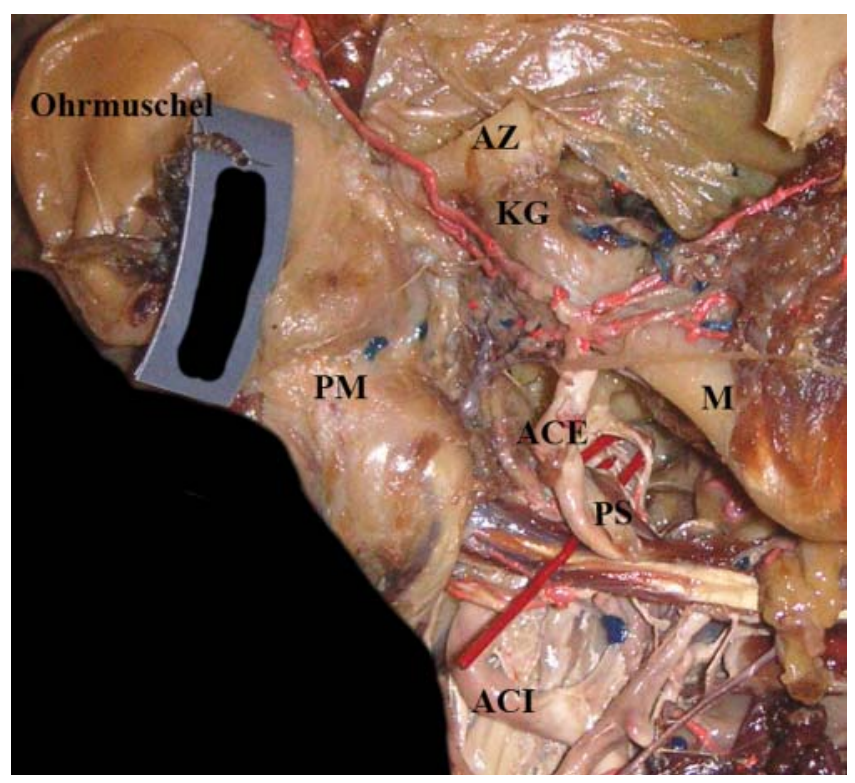

Gruppe $\mathrm{C}$ mit fehlender FSP. Ansicht einer rechten Gesichtshälfte: Als Orientierung dienen die Mandibula $(M)$, der Processus mastoideus (PM), Processus styloideus $(P S)$ und das Kiefergelenk (KG). Der Arcus zygomaticus (AZ) ist knapp vor dem KG abgeschnitten. Die rote Sonde ist medial vom PS nach kranial geschoben und deutlich sichtbar

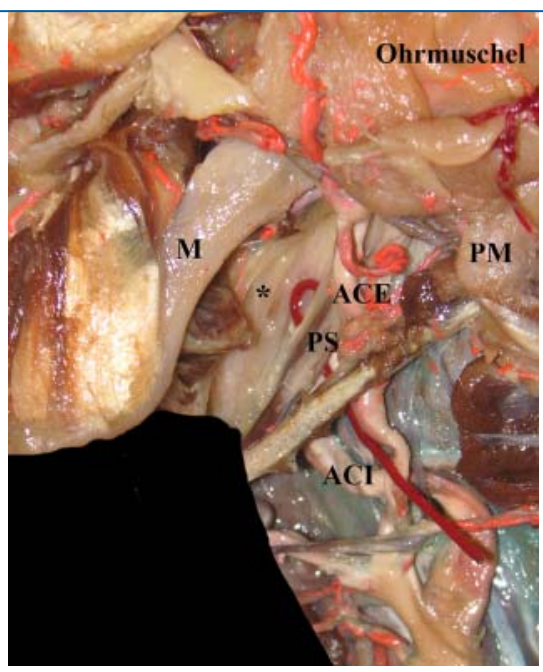

Abb. 5 - Beispiel für einen Fall der Gruppe B an einer linken Gesichtshälfte: Die FSP weist im kaudalen Bereich eine Perforation auf. Die Sonde ist durch die Schwachstelle geschoben. Beschrieben ist hier zusätzlich die A. carotis externa $(A C E)$ lateral der Faszie ${ }^{*}$ ) und zur weiteren Orientierung die Ohrmuschel

Schmerzreduktionsbreite sehr stark variieren. Elsner [4] berichtet in einer retrospektiven Studie sowohl von einer schmerzverstärkenden Wirkung bis hin zu 99\% Schmerzreduktion, bzgl. der initialen Wirkung der GLOA von einer Schmerzlinderung bei $77 \%$ der analysierten Fälle. Die mittelfristige Wirkung betrachtend berichtet Elsner [4] von einer Schmerzreduktion in $66 \%$ der Fälle. Richter [13] untersuchte die GLOA des GCS bei 32 Patienten, wobei der N. V/3 in $78,1 \%$ der Fälle einzeln oder in Verbindung mit anderen Trigeminusstämmen betroffen war. Sie nennt eine Schmerzfreiheit bei 18 der 32 Patienten, inkomplette Remissionen in 10 Fällen und unveränderte Schmerzsituation bei 4 Patienten. Jedoch fehlt in den Ergebnissen leider die therapeutische Wirkung bezo- gen auf die betroffenen Stämme, die für die vorliegende Arbeit von großem Interesse gewesen wäre.

Spacek et al. [16] berichten über eine gute Schmerzreduktion bei 21 Patienten, die mittels GLOA am GCS therapiert wurden. Evaluiert wurde dabei anhand der „visual analogue scale“. In einer anderen Studie berichten sie von über $37 \%$ Ausfall bei alleiniger Therapie durch GLOA am GCS [17]. Zusätzlich ist in dieser Arbeit eine 2. Blockadetechnik, die Blockade des Ganglion spheno- oder auch pterygopalatinum, mit einbezogen. Diese 2. Technik zielt eigentlich auf den 2 . Hauptast des N. trigeminus: den N. maxillaris. Leider finden sich in der letztgenannten Arbeit von Spacek keinerlei genauere Daten bzgl. der Anzahl der GLOA in Relation zur Misserfolgsquote.
Feigl et al. [7] weisen auf die FSP als mögliche Barriere und somit als Ursache für eine geringere Erfolgsquote der Schmerzreduktion hin. Doch werden sowohl von Feigl et al. als auch in der Literatur keinerlei Hinweise auf die Häufigkeit und Ausbildung dieser Faszie gegeben. Dies ist nun durch diese genauere Untersuchung beschrieben. Das Ergebnis von $83,6 \%$ vollständig ausgebildeter Faszien belegt, dass die Faszie bei Blockade des GCS und Einhaltung der originalen Stichrichtung von Pejic [12] die Ausbreitung des Opiats bis in die Fossa infratemporalis behindert und somit eine zusätzliche Opioidwirkung des 3. und eventuell 2. Trigeminusastes verhindert. Schlussfolgernd kann die Faszie Auswirkungen auf die Erfolgsrate der Schmerzreduktion haben. Eine Umgehung der Faszie wäre nur durch eine nach weiter vorne verlegte Einstichstelle möglich, durch die die Nadelspitze evtl. in den vor der Faszie gelegenen Raum gelangen könnte. Jedoch würde dann die bewiesene adjuvante Schmerzreduktion mittels der GLOA am GCS wegfallen $[4,13,16,17]$.

Schlussendlich kann sich bei fehlender Faszie noch ein weiteres Hindernis in den Weg stellen: das Lig. sphenomandibulare (LSM). Dieses Band wurde von Garg u. Townsend [9] im Rahmen einer zahnärztlichen Untersuchung an 7 Leichen genannt, welches die Ausbreitung von Anäs- 
thetika zum N. alveolaris inferior behindern kann. Die beiden Autoren beschreiben sehr variabel ausgebildete Bänder, von dünner bis breiter ligamentärer Struktur. Im Rahmen unserer Untersuchung beobachteten wir in fast allen Fällen breite bandartige Strukturen und komplett ausgebildete Faszienplatten aponeurotischen Charakters in 10 Fällen.

\section{Fazit für die Praxis}

Auf der Suche nach größter Effizienz von Schmerztherapien müssen Blockadetechniken immer wieder evaluiert werden. Dabei sollte auch die Suche nach möglichen Ursachen für eine variierende Schmerzreduktion vorangetrieben werden. In dieser Arbeit wird aufgezeigt, dass eine vollausgebildete FSP den positiven Nebeneffekt der zusätzlichen Opioidwirkung am N. V/3 bei einer GLOA verhindern kann. In über $83 \%$ der Fälle stellt sich die FSP vollausgebildet als unüberwindbare Wand in den Weg. Weiter wird eine im Laufe der Jahrzehnte unter den Tisch gekehrte Faszie in Erinnerung zurückgerufen, die trotz ihres mystischen Daseins sich als schwerwiegendes Hindernis aufbaut. Wir hoffen, mit dieser Untersuchung einen Beitrag zur Ursachenforschung bezüglich variierender Schmerzreduktionsquoten bei der Therapie der TN leisten zu können.

\section{Korrespondierender Autor}

\section{Dr. G. Feigl}

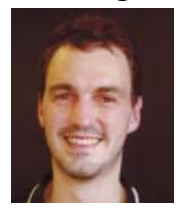

Division für Anatomie im Department für Morphologie, CMU der Universität Genf Rue Michel Servet 1, 1211 Genf Georg.Feigl@medecine.unige.ch

Interessenkonflikt. Es besteht kein Interessenkonflikt. Der korrespondierende Autor versichert, dass keine Verbindungen mit einer Firma, deren Produkt in dem Artikel genannt ist, oder einer Firma, die ein Konkurrenzprodukt vertreibt, bestehen. Die Präsentation des Themas ist unabhängig und die Darstellung der Inhalte produktneutral.
2. Corning HK (1917) Topographie des Pharynx. In: Corning HK (Hrsg) Lehrbuch der topographischen Anatomie. Bergmann, Wiesbaden, S 131

3. Eisler P (1912) Fascia cervicalis. In: von Lanz T, Wachsmuth W (Hrsg) Praktische Anatomie: Kopf. Springer, Berlin Heidelberg New York, S 382

4. Elsner F, Radbruch L, Gaertner J et al. (2006) Opoidwirksamkeit am Ganglion cervicale superius bei neuropathischen Schmerzen im Kopf- und Gesichtsbereich. Schmerz 20: 268-276

5. Fanghänel J (2003) Kopf, Cranium, und Hals, Collum. In: Fanghänel J, Pera F, Anderhuber F, Nitsch $\mathrm{R}$ (Hrsg) Waldeyer - Anatomie des Menschen, 17. Aufl. de Gruyter, Berlin, S 177-353

6. Federative Committee on Anatomical Terminology (1998) Terminologia Anatomica. Thieme, Stuttgart New York

7. Feigl G, Rosmarin W, Likar R (2006) Blockade des Ganglion cervicale superius des Truncus sympathicus: Warum es nicht immer geht! Schmerz 20: 277-284

8. Frick H (1987) Bindegewebsräume des Halses, Einbau der Leitungsbahnen und Organe. In: Frick H, Leonhardt H, Starck D (Hrsg) Spezielle Anatomie I. Thieme, Stuttgart New York S 738

9. Garg A, Townsend G (2001) Anatomical variation of the sphenomandibular ligament. Aust Endod J 27(1): 22-24

10. Hafferl A (1969) Das Spatium parapharyngeum. In: Hafferl A (Hrsg) Lehrbuch der topographischen Anatomie. Springer, Berlin Heidelberg New York, S 195-200

11. Leonhardt H (1988) Halsteil des Truncus sympathicus. In: Rauber A, Kopsch F (Hrsg) Anatomie des Menschen, Bd IV. Thieme, Stuttgart New York, S 17 und 134

12. Pejič S (1965) Transoral block of the cervico- endocranial sympathetic system in otorhinolaryngology. Srpski Arhiv Za Celokupno Lekarstvo 93(7): 719-730

13. Richter M (1997) Ganglionäre lokale Opioidanalgesie am Ganglion cervicale superius zur Behandlung der Trigeminusneuralgie. Dissertationsschrift an der Julius-Maximilians Universität Würzburg

14. Rouvière H (1925) Région pharyngienne. In: Masson et al. (eds) Précis d'Anatomie et de Dissection. Libraires de l'Academie de Médicine: 177

15. Schiebler T (1997) Die Leitungsbahnen am Kopf und Hals. In: Schiebler T, Schmidt W, Zilles K (Hrsg) Anatomie, 7. Aufl. Springer, Berlin Heidelberg New York, $\mathrm{S} 463$

16. Spacek A, Böhm D, Kress HG (1997) Ganglionic local opioid analgesia for refractory trigeminal neuralgia. Lancet 349: 1521

17. Spacek A, Hanl G, Groiss O et al. (1998) Akupunktur und ganglionäre lokale Opoidanalgesie (GLOA) bei Trigeminusneuralgie. Wien Med Wochenschr 19: 447-449

18. Thiel W (1992a) Die Konservierung ganzer Leichen in natürlichen Farben. Ann Anat 174: 185-195
Stefan Engel-Wissenschaftspreis 2006

Schmerzteam aus Datteln für stationäre Kinderschmerztherapie mit der Stefan Engel- Medaille 2006 ausgezeichnet.

Etwa 250.000 Kinder in Deutschland leiden an chronischen Kopf-, Bauch- und Rückenschmerzen, Tumorschmerzen, rheumatischen Schmerzen oder anderen Schmerzen. Wenn ein Kind wegen dauernder starker Schmerzen häufig nicht zur Schule gehen kann und wenn ambulante Behandlungsangebote nicht mehr ausreichen, greift das Dattelner Stationäre Schmerzkonzept.

Die Spezialisten der Vestischen Kinderund Jugendklinik Datteln, einer kooperierenden Klinik der Universität Witten/Herdecke, haben ein dreiwöchiges, stationäres Therapieprogramm entwickelt, in das auch die Familien der betroffenen Kinder einbezogen sind. Eine Befragung der Patienten bis zu zwei Jahre nach ihrer Entlassung zeigte jetzt, dass der Ansatz wirkt: Die Schmerzstärke sinkt dauerhaft, das subjektive Wohlbefinden steigt, Schulfehlzeiten gehen zurück.

Diese Ergebnisse überzeugten auch die Juroren des Stefan Engel Wissenschaftspreis 2006 der Deutschen Gesellschaft für Sozialpädiatrie und Jugendmedizin. Anlässlich ihrer 58. Jahrestagung in Mainz verliehen sie die Stefan Engel Medaille und Stefan Engel Preis an das Schmerzteam aus Datteln.

Quelle: Vestische Kinder- und Jugendklinik, Universität Witten/Herdecke, www.kinderklinik-datteln.de

\section{Literatur}

1. Barry KB (2005) Pharynx. In: Standring S (ed) Gray's anatomy, 39th edn. Elsevier, Edinburgh London New York, pp 619-631 


\section{Hier steht eine Anzeige.}

黛 Springer 